\title{
Gestão do capital intelectual nos Correios
}

\section{Management of intellectual capital at the Post Office}

\section{Recebimento: 11/12/2020 - Aceite: 1501/2021 - Publicação: 01/02/2021 \\ Processo de Avaliação: Double Blind Review}

\author{
Leonardo Fabris Lugoboni ${ }^{1}$ \\ Doutor em Administração pela Universidade São Paulo (USP) \\ Professor do Mestrado da Universidade Ibirapuera e do Centro Universitário Alves Faria \\ leonardo.lugoboni@gmail.com
}

Moyses Vieira da Silva

Especialista em Controladoria pela Fundação Escola do Comércio Álvares Penteado (FECAP)

moysesconta@hotmail.com

Marcus Vinicius Moreira Zittei

Doutor em Ciências Contábeis e Administração pela Fundação Universidade Regional de Blumenau (FURB)

Professor e Coordenador do Mestrado em Governança do Centro Universitário das Faculdade Metropolitanas Unidas (FMU)

marcuszittei@zittei.com.br

José Orcelio do Nascimento

Mestre em Ciências Contábeis pela Fundação Escola do Comércio Álvares Penteado (FECAP)

Professor e Coordenador da Especialização em Gestão Pública da Fundação Escola do Comércio Álvares Penteado (FECAP)

jorcelio@uol.com.br

Flávia Cruz de Souza Murcia

Doutora em Administração pela Universidade Federal de Santa Catarina (UFSC)

Professor do Mestrado Profissional em Administração da Universidade Ibirapuera (UNIB)

flavia.murcia@ibirapuera.edu.br

\footnotetext{
${ }^{1}$ Autor para correspondência: Universidade Ibirapuera (UNIB): Av. Interlagos, 1329- São Paulo- SP, CEP 04661-100, Brasil
} 


\section{RESUMO}

Este estudo teve como objetivo evidenciar como os gerentes dos Correios gerem seu capital intelectual. Optou-se pela realização de um estudo em duas etapas: análise documental e pesquisa survey. Na primeira etapa, foram identificados pontos fortes no que se refere à gestão do capital intelectual dos Correios: plano de cargos e salários ajustado com a política de desenvolvimento profissional, criação do programa Inova Correios, e identificação das necessidades e expectativas dos clientes. Na segunda etapa, 37 gerentes de agências da cidade de São Paulo destacaram a importância de alguns indicadores relacionados às três dimensões do capital intelectual: satisfação do funcionário, eficiência nos processos, qualidade nos processos, clientes dos Correios e gestão da carteira de clientes. Ressalta-se a necessidade de melhoria em diversos indicadores por parte da empresa, além da implantação de ferramentas para controle e mensuração do capital intelectual, com o intuito de agregação de valor e continuidade do negócio.

Palavras-chave: capital intelectual, mensuração, capital humano, capital estrutural, capital relacional.

\section{ABSTRACT}

This study aimed to show how post offices managers manage their intellectual capital. It was decided to carry out a two-stage study: documentary analysis and research survey. In the first stage, strengths were identified about the management of intellectual capital of the post offices: a job and salary plan adjusted with the professional development policy, creation of the Inova post offices program, and identification of customer needs and expectations. In the second stage, 37 agency managers from the city of São Paulo highlighted the importance of some indicators related to the three dimensions of intellectual capital: employee satisfaction, process efficiency, quality of processes, post offices customers and client portfolio management. The need for improvement in several indicators by the company is emphasized, as well as the implementation of tools to control and measure intellectual capital, with the purpose of aggregating value and business continuity.

Keywords: intellectual capital, measurement, human capital, structural capital, relationship capital. 


\section{INTRODUÇÃO}

A valorização das empresas atualmente não se limita apenas ao seu valor contábil ou ao valor de seus bens tangíveis. De acordo com Matos, Reina, Ensslin e Reina, (2011), o capital intelectual é o meio mais completo para valorizar as organizações, diante da competição existente nos negócios.

Conforme Antunes e Martins (2002), o grupo Skandia montou um modelo para medir o capital intelectual - orientando-se pela diferença entre o valor de mercado e o seu valor contábil, ajudando os gestores das empresas nas decisões de investimento acerca dos componentes que fazem parte desse capital.

O setor postal tem sofrido uma forte pressão nas últimas décadas devido ao desenvolvimento de novas tecnologias de comunicação e informação. Assim, os Correios estão se revolucionando para continuar cumprindo seu papel secular e, diante desse novo panorama. Os Correios implantaram o Modelo de Excelência da Gestão da Fundação Nacional da Qualidade e tem como objetivo transformar-se em uma empresa orientada pelo conhecimento.

Assim, o presente estudo procurou responder à seguinte questão de pesquisa: como os Correios realizam a gestão do capital intelectual? Foi elaborado, então, o seguinte objetivo geral: evidenciar como os gerentes dos Correios gerem seu capital intelectual.

Segundo Gracioli, Godoy, Lorenzett e Godoy (2012) o capital intelectual é cada vez mais importante para a sobrevivência das empresas. Dessa forma, a inovação da empresa, com novos produtos e serviços, só é possível através do capital intelectual, ajustando a empresa às constantes mudanças do mercado.

Esse estudo possibilitará aos gestores dos Correios a percepção sobre a adequação do método utilizado para mensurar o capital intelectual à necessidade da empresa perante o mercado. Por consequência, os resultados gerados serão importantes para a empresa e diversos stakeholders. 


\subsection{Capital Intelectual}

Conforme Wernke, Lembeck e Bornia, (2003) o precursor na valorização do conhecimento foi Peter Drucker com seu livro Sociedade Pós Capitalista, onde citou o término da fase industrial e o começo da fase do conhecimento.

Na figura 1 são apresentados alguns conceitos de capital intelectual:

\begin{tabular}{|l|l|}
\hline $\begin{array}{l}\text { Edvinsson } \\
\text { Malone (1998) }\end{array}$ & e $\begin{array}{l}\text { Corresponde ao capital intelectual, parte dinâmica oculta que sustenta os ativos tangíveis } \\
\text { da empresa. }\end{array}$ \\
\hline Stewart (1998) & $\begin{array}{l}\text { Definiu o capital intelectual como a soma de todos os conhecimentos que os funcionários } \\
\text { da empresa possuem e que dão a esta uma vantagem competitiva. }\end{array}$ \\
\hline Lev (2001) & $\begin{array}{l}\text { Um ativo intangível que, se gerenciado com sucesso, proporciona benefícios futuros para } \\
\text { a empresa. }\end{array}$ \\
\hline
\end{tabular}

Figura 1 - Conceitos de Capital Intelectual

Fonte: Adaptado de Silva Filho, Holanda e Rocha (2006)

Edvinsson e Malone (1998) retratam o capital intelectual da seguinte maneira:

Capital intelectual $=$ Capital humano + capital estrutural

O capital estrutural é composto pela soma do capital de inovação, capital de processos e capital de relacionamento, de acordo com a Figura 2:

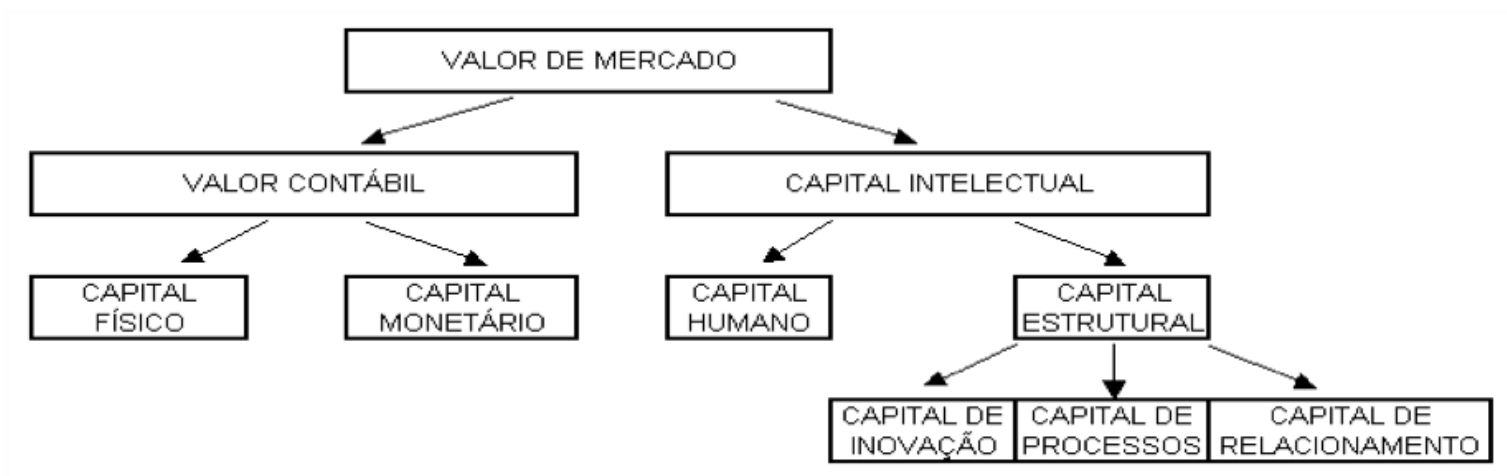

Figura 2 - Composição do Valor de Mercado Fonte: Edvinsson e Malone (1998)

Os mesmos autores detalham a composição do capital intelectual (Figura 3), a qual será mais bem detalhada nas próximas subseções do estudo: 


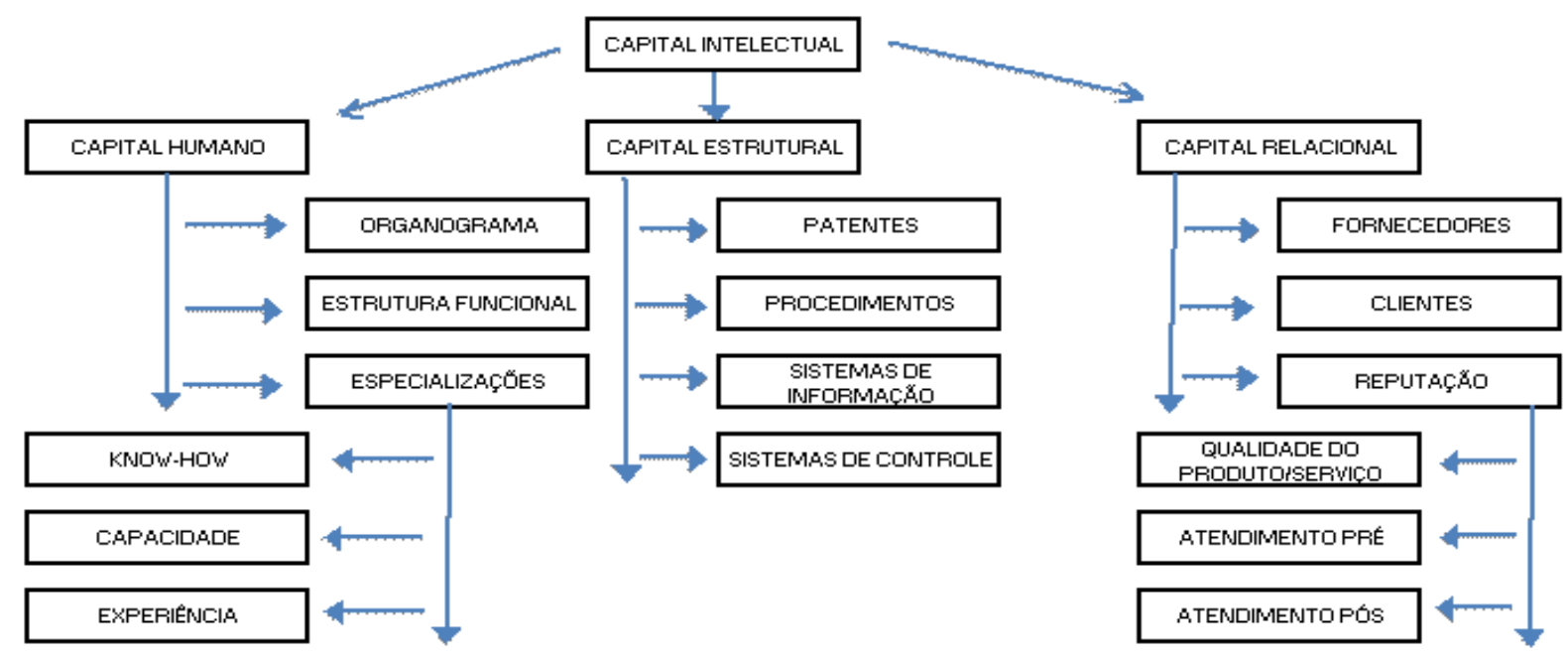

Figura 3 - Composição do Capital Intelectual Fonte: Edvinsson e Malone (1998)

\subsubsection{Capital Humano}

Conforme Carson, Ranzijn, Winefield \& Marsden (2004), o capital humano é definido como o potencial das pessoas que servem como fonte de inovação e renovação nas organizações.

De acordo com Rezende, Avila e Maia e (2012), os ativos intangíveis são divididos na competência dos funcionários, estrutura interna e estrutura externa. Dessa forma, é impossível existir uma organização sem pessoas e, mesmo estas não pertencendo ao patrimônio das empresas, são consideradas ativos, pois através de seus conhecimentos, habilidades e experiências podem desenvolver tanto ativos tangíveis quanto intangíveis.

\subsubsection{Capital Estrutural}

Conforme Bontis (1998) e Khan (2011), o capital estrutural gerencia os processos da empresa podendo auxiliar os funcionários no desenvolvimento do correto desempenho intelectual, bem como na performance da empresa como um todo.

Segundo Arnosti, Neumann, Oliveira e Yomura, (2009), o capital estrutural de uma organização pode ser mensurado. Assim, pode fazer parte do patrimônio da empresa e, consequentemente, ser vendido, o que não ocorre com o capital humano. 


\subsubsection{Capital de Relacionamento}

O capital de relacionamento significa o valor agregado na relação da empresa com os agentes do ambiente externo formado pelos acionistas, fornecedores, credores, clientes e outros (Arnosti, Neumann, Oliveira \& Yomura, 2009).

Conforme Oliveira (1999) e Gibbert, Leibold e Voelpel (2001), assim como a organização investe em seu corpo funcional com o objetivo de aumentar o valor do capital intelectual, também é necessário investimento nos clientes, formando um intangível comum entre as partes e agregando valor adicionado em toda cadeia produtiva

\subsection{Modelos de Mensuração do Capital Intelectual}

A mensuração do capital intelectual ainda é uma tarefa difícil para os profissionais da área contábil. De acordo com Iudícibus (1997), em boa parte das situações as demonstrações contábeis omitem no seu ativo o valor do capital intelectual, havendo um atraso na contabilidade. $\mathrm{O}$ mesmo autor ressalta que, para qualquer item ser considerado ativo, este deve atender a quatro requisitos básicos:

1. Ser considerado bem ou direito para a empresa;

2. Poder mensurá-lo monetariamente;

3. A empresa tem que ter sua propriedade, posse ou controle a longo prazo, e

4. Ter perspectivas de que o bem traga benefícios futuros.

A seguir são detalhados modelos de gestão que auxiliam os administradores na mensuração do capital intelectual.

\subsubsection{Balanced Scorecard (BSC)}

O Balanced Scorecard (BSC) é criação de Kaplan e Norton (1992), foi resultado da busca por ferramentas de mensuração da performance das empresas e ao longo dos anos o seu uso tem sido crescente. 


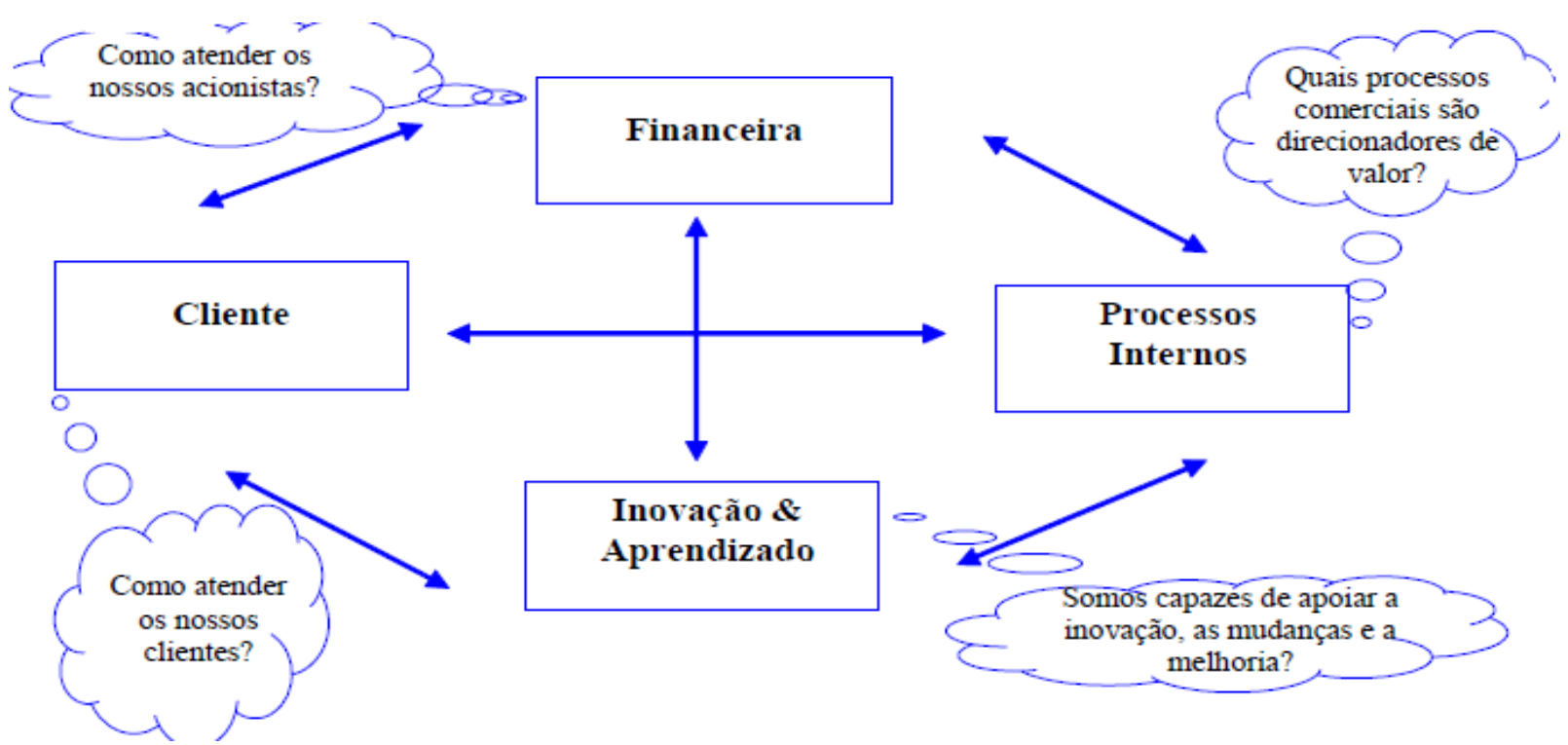

Figura 4 - As Quatro Perspectivas do BSC Fonte: Kaplan e Norton (1992).

Segundo Olve, Roy e Wetter (1999), o BSC mensura diversas circunstâncias e condições que são fundamentais para a empresa. Contudo, os autores enfatizam que a mensuração por meio do BSC não é o mais importante, mas sim a discussão de ideias sobre as medidas no passado, presente e futuro.

\subsubsection{Navegador Skandia}

O Navegador Skandia é formado por cinco focos: humano, financeiro, cliente, processo, renovação e desenvolvimento. De acordo com Edvinsson e Malone (1998), a empresa direciona suas forças para esses focos e a partir deles desenvolve o seu capital intectual no ambiente de concorrência.

Segundo Bollen, Vergauwen \& Schnieders, (2005), a Skandia relacionou o termo capital intelectual com o ativo intangível e publicou o primeiro relatório em 1995 sobre o tema. A partir daí outras empresas seguiram o exemplo da Skandia e se tornaram conscientes da necessidade da liderança e do crescimento do capital humano para aumentar o potencial da cultura empresarial como fundamental recurso para vantagem competitiva sustentável. 


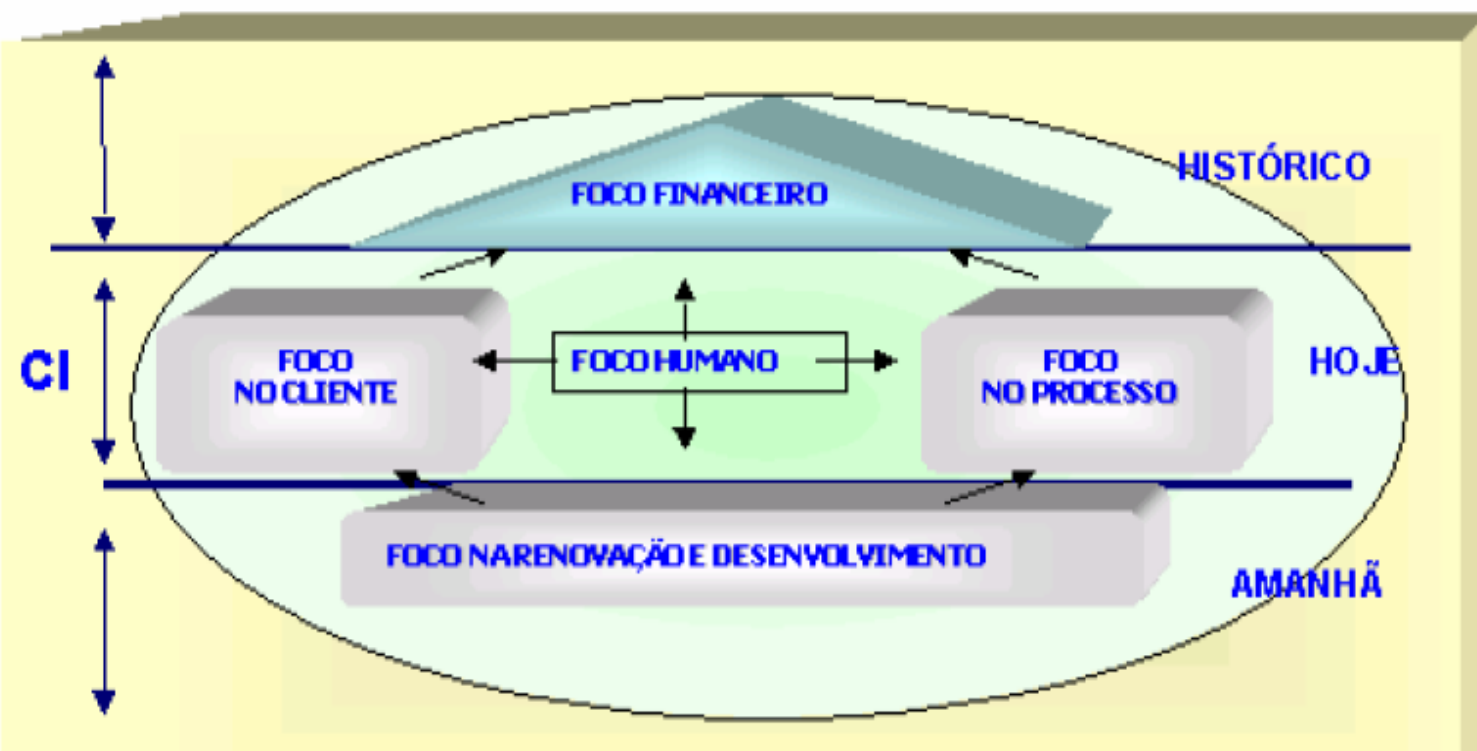

AMBIENTE OPERACIONAL

Figura 5 - Modelo Skandia

Fonte: Edvinsson e Malone (1998)

Ainda de acordo com Edvinsson e Malone (1998) e Dumay, Rooney e Marini (2013), o Foco na Renovação e Desenvolvimento faz menção ao futuro da empresa, outra parte do capital estrutural da empresa.

\subsubsection{Prêmio Nacional de Qualidade (PNQ)}

Conforme a Fundação Nacional da Qualidade (FNQ) durante a década de 80, a liderança industrial americana foi abalada devido à qualidade da produção japonesa. Assim, a partir deste acontecimento, pesquisadores analisaram várias empresas de sucesso com características similares entre elas, mas que as distinguiam das demais. Os resultados desta pesquisa deram origem em 1987 aos critérios de excelência do Prêmio Malcolm Baldrige. Em 1991, por sua vez, uma fundação sem fins lucrativos, promoveu o Prêmio Nacional da Qualidade (FNQ, 2007).

O PNQ contribui para a mensuração do capital intelectual pois absorve a administração da empresa na sua totalidade, a qual possui como missão cumprir o planejamento estratégico através da satisfação das necessidades das partes interessadas (FNQ, 2007).

O modelo de gestão do PNQ é composto por oito critérios de excelência: Liderança, Estratégias e Planos, Clientes, Sociedade, Informações e Conhecimento, Pessoas, Processos, e Resultados (FNQ, 2007). 


\subsection{Setor Postal}

Desde a década de 80 , o setor postal tem sido revolucionado, sobretudo pela visão empresarial que passou a imperar nos negócios, pelas novas tecnologias de comunicação, pelos processos de automação e mecanização, pela internacionalização dos negócios, pela entrada de operadores privados em todos os eventos da cadeia do negócio postal e pelo desenvolvimento do setor de transporte, sobretudo na Europa. O novo século trouxe consigo a inauguração de uma nova era para o setor.

Esse processo de interpenetração de setores sinaliza o aumento crescente da complexidade para a gestão dos negócios postais, pois cada vez mais será preciso entender, concomitantemente, o funcionamento de vários setores da economia, acompanhar as suas legislações específicas, os seus fóruns decisórios, cenários etc.

\subsection{Estudos Anteriores}

Khalique, Bontis, Shaari e Isa, (2015) avaliaram a relação entre sub componentes do capital intelectual (capital humano, capital de clientes, capital estrutural, capital social, capital tecnológico e capital espiritual) e performance organizacional em pequenas e médias empresas que atuam no setor de eletrônicos no Paquistão. Com a utilização de regressão múltipla, os resultados indicaram que os sub componentes apresentaram significância positiva sobre a performance das empresas, exceto a variável 'capital humano', o que é inesperado na medida em que este componente é considerado um dos mais críticos do capital intelectual.

Ramírez, Manzaneque e Priego (2015) elaboraram um modelo de avaliação do capital intelectual (capital humano, capital estrutural e capital relacional) em universidades públicas espanholas composto por elementos intangíveis e seus respectivos indicadores, os quais totalizaram 31. Tais indicadores foram validados por meio de entrevistas com membros dos Conselhos Sociais das universidades que compuseram a amostra, chegando a um total de 13 indicadores-chave.

Andreeva e Garanina (2016) analisaram a relação entre os três principais elementos de capital intelectual (humano, relacional e estrutural) e a performance de 240 organizações russas. As autoras utilizaram análise fatorial e regressão múltipla e os resultados demonstraram que os capitais humano e estrutural influenciam positivamente na performance.

Secundo, Dumay, Schiuma e Passiante (2016) realizaram um estudo exploratório com o intuito de elaborar uma estrutura conceitual para gestão do capital intelectual em 
universidades considerando a perspectiva da inteligência coletiva, uma vez que varios stakeholders contribuíram para a sua construção.

Smriti e Das (2018) examinaram o efeito do capital intelectual na performance financeira de empresas indianas. Os resultados indicaram que o capital humano possui o maior impacto na produtividades das companhias analisadas. Além disso, observou-se que a eficiência do capital estrutural e do capital empregado foram igualmente importantes para o crescimento das vendas e do valor de mercado das empresas.

\section{METODOLOGIA}

Quanto aos objetivos, esta pesquisa é descritiva e de acordo Andrade (2010), nesse tipo de pesquisa não há interferência ou manipulação dos fenômenos pelo pesquisador, mas sim a observação, o registro, a análise, a classificação e a interpretação dos fatos.

Quanto aos procedimentos, divide-se em: documental e survey. Na pesquisa documental, busca descrever os indicadores e relatórios presentes na gestão atual dos Correios. Para Bervian, Cervo e Da Silva (2007), na pesquisa documental são analisados documentos com o objetivo de demonstrar e fazer analogia de características importantes. $\mathrm{Na}$ pesquisa survey, foram distribuídos questionários aos gestores das agências de Correios com o intuito de verificar, sob a análise das três dimensões do capital intelectual - capital humano, capital estrutural e capital relacional - qual a ótica deles com relação ao uso dos indicadores de mensuração do capital intelectual em suas unidades. De acordo com Andrade (2010), o survey é formado por perguntas em que o informante responde, independente da presença do pesquisador.

Com o objetivo de consolidar a literatura para embasar as duas etapas da pesquisa, elaborou-se a Figura 6 relacionando cada aspecto e seu respectivo autor. 


\begin{tabular}{|c|c|}
\hline \multicolumn{2}{|l|}{ Aspectos avaliados por meio de indicadores de desempenho } \\
\hline Aspectos Relacionados ao Capital Humano & Autores \\
\hline Clima e satisfação do funcionário & Vieira, Appio, \& Slongo (2007) \\
\hline Políticas de desenvolvimento dos funcionários & Bundchen e Silva (2005) \\
\hline Liderança e delegação de responsabilidades & $\begin{array}{l}\text { Kich, Pereira, Emmendoerfer \& Santos, } \\
(2008)\end{array}$ \\
\hline Inovação & Fontenelle (2012) \\
\hline Desligamento de funcionários & Silva, Basques, \& Facco, (2006) \\
\hline Habilidades desenvolvidas valorizadas & $\begin{array}{|lccc|}\begin{array}{l}\text { Demo, Fogaça, } \\
\text { Francischeto(2011) }\end{array} & & & \\
\end{array}$ \\
\hline Aspectos Relacionados ao Capital Estrutural & Autores \\
\hline Questões econômicas e financeiras vinculadas ao capital intelectual & $\begin{array}{l}\text { Vital, Cavalcanti, Dalló, Moritz e Costa, } \\
(2009)\end{array}$ \\
\hline Capacidade de atendimento & Silva,et al. (2006) \\
\hline Estrutura organizacional & Dias Junior, Lima e Dalmau (2011) \\
\hline Gestão dos procedimentos & Schneider e Moretto (2006) \\
\hline Eficiência nos processos & Estrada e Almeida, (2007) \\
\hline Qualidade nos processos & $\begin{array}{l}\text { Souza, Guerra, Lara, Gomide, Pereira e } \\
\text { Freitas (2009) }\end{array}$ \\
\hline Sociedade em geral & Reis e Giacomini (2008) \\
\hline Meio ambiente & Krücken-Pereira, Abreu e Bolzan (2002) \\
\hline Aspectos Relacionados ao Capital Relacional & Autores \\
\hline Clientes dos Correios & Piva, Fumagalli, Baptista \& da Silva (2007) \\
\hline Qualidade do relacionamento & Lima, e Baptista (2013) \\
\hline Gestão da carteira & Rozzett e Demo (2010) \\
\hline Participação de mercado & Scherer e Gomes (2010) \\
\hline Quantidade de agências & Vasconcelos (2010) \\
\hline Análise da concorrência & $\begin{array}{l}\text { Contador, Contador, Carvalho, e Contador, } \\
(2006)\end{array}$ \\
\hline Canais de distribuição & Gião e Oliveira (2009) \\
\hline Despesas de atendimento por clientes & Piva et al. (2007) \\
\hline
\end{tabular}

Figura 6 - Indicadores de Mensuração do Capital Intelectual

Fonte: Elaborado pelos autores

\section{RESULTADOS}

\subsection{Análise Documental}

A análise documental foi embasada nas informações do resultado da Autoavaliação Assistida realizada pela Fundação Nacional da Qualidade (FNQ) - órgão que auxilia os Correios na implementação do Modelo de Excelência da Gestão (MEG) -, evidenciando os resultados dos itens que envolvem o capital intelectual (Capital Humano, Capital Estrutural e Capital Relacional). Além disso, foram analisados documentos gerenciais, relatórios técnicos e pesquisas nos sites das áreas envolvidas, através da intranet da empresa. 


\subsubsection{Capital Humano nos Correios}

Diversos pontos fortes relacionados ao Capital Humano foram evidenciados e merecem destaque. Dentre eles, menciona-se:

a. Organização de trabalho - plano de cargos, carreiras e salários colabora para a política de desenvolvimento e crescimento funcional e salarial;

b. Seleção e contratação de pessoas - a admissão de funcionários do quadro fixo é vinculada às normas que regem as empresas públicas;

c. Integração de funcionários recém contratados - por meio da matriz de capacitação, o funcionário é treinado antes de ocupar o cargo;

d. Avaliação de desempenho - as competências profissionais exigidas pela empresa são avaliadas através do Gerenciamento de Competências e Resultados (GCR);

e. Remuneração, reconhecimento e incentivos - os funcionários que forem bem avaliados no GCR são elegíveis para participação de lucros, campanhas de incentivos a vendas e promoção por mérito.

Por outro lado, também foram verificadas oportunidades de melhorias no Capital Humano dos Correios, como verifica-se a seguir:

a. Dimensionamento do quadro de pessoal - não há evidência de critério formalizado para dimensionamento do quadro de pessoal dos processos de apoio;

b. Processo de seleção e contratação de pessoas - não é possível avaliar a efetividade do processo de seleção e contratação de pessoas, pois não foram apresentadas análises qualitativas e quantitativas que permitam comprovar a consonância com as estratégias dos Correios;

c. Avaliação de desempenho das equipes - não há evidência de prática para a avaliação de desempenho das equipes nos processos de apoio;

d. Obtenção de metas de alto desempenho - não é possível garantir que o GCR estimule a obtenção de metas de alto desempenho, pois não há análise sistematizada do alinhamento entre as metas gerenciadas no GCR e as metas registradas no plano de metas regional.

\subsubsection{Capital Estrutural nos Correios}

No que se refere ao Capital Estrutural dos Correios, foram observados os seguintes pontos fortes: 
a. Desenvolvimento de novos produtos - para a formatação de novos produtos e serviços são utilizadas técnicas e ferramentas como matriz Ansoff, matriz BCG e mapeamento de processos;

b. Processos principais do negócio e processos de apoio - os departamentos operacionais da empresa planejam a criação e o desmembramento das unidades distribuidoras, prevendo o aumento na demanda de encomendas;

c. Avaliação do potencial de idéias criativas - o programa Inova Correios é controlado pelo departamento de inovação, que emite relatórios de participação por unidades e diretorias regionais;

d. Atendimento dos requisitos dos processos da empresa - o controle dos processos principais do negócio é realizado por meio do monitoramento dos indicadores de desempenho e qualidade operacional;

e. Análise e melhoria dos produtos - os principais processos operacionais do negócio (tratamento e distribuição) são analisados e melhorados em dois períodos distintos no ano: no fórum de revisão para os seus manuais operacionais e no fórum de revisão da padronização do processo produtivo.

Também foram constatadas oportunidades de melhorias no Capital Estrutural dos Correios, como relatado a seguir:

a. Principais requisitos e indicadores de desempenho dos processos - não estão definidos os principais requisitos e os respectivos indicadores de desempenho dos processos de apoio de contratação e gestão de contratos, suprimentos, jurídico, relacionamento institucional e comunicação;

b. Ideias criativas convertendo-se em inovações - não foram apresentadas informações qualitativas ou quantitativas que comprovem que o programa Inova Correios seja eficaz para conversão de ideias em inovação em produtos ou processos;

c. Atendimento dos requisitos aplicáveis aos processos - não há como garantir a padronização dos requisitos do processo de atendimento nas agências terceirizadas;

d. Investigação de característica de produtos e processos - a prática de benchmarking de processos não está sistematizada. Não estão definidos padrão de trabalho, responsabilidades nem indicadores de desempenho para o processo;

e. Tratamento de não conformidade - não há como garantir tratamento das ocorrências e implementação das respectivas ações corretivas identificadas no sistema de 
acompanhamento da padronização do processo produtivo e padronização e melhoria do atendimento.

\subsubsection{Capital Relacional nos Correios}

O Capital Relacional dos Correios é determinado pelos seguintes pontos fortes:

a. Segmentação de mercado - o processo de segmentação e categorização de clientes está manualizado e tem como objetivo agrupar os clientes em segmentos e categorias, de acordo com o valor que representam para os Correios: estratégico, corporativo, empresarial, comercial e varejo;

b. Identificação das necessidades e expectativas dos clientes - as principais formas de captura são: pesquisa de imagem, pesquisa com clientes estratégicos e corporativos, pesquisa de produtos e serviços e pesquisa concorrencial e análise de mercado;

c. Divulgação dos produtos - o plano anual de comunicação analisa as peculiaridades e necessidades de comunicação de cada área com base no plano estratégico;

d. Comunicação da marca - guia de comunicação visual enfatiza a aplicação gráfica da marca.

Contudo, observou-se oportunidades de melhoria no Capital Relacional dos Correios, como exposto a seguir:

a. Critérios para definição de clientes-alvo - não estão formalizados os critérios adotados para a definição dos clientes-alvo dentro de cada um dos segmentos de clientes;

b. Identificação das necessidades e expectativas dos clientes - as práticas utilizadas para a identificação de necessidades e expectativas dos clientes, pesquisas e agentes de relacionamento não abrangem os clientes potenciais e ex-clientes;

c. Divulgação da marca e dos produtos - não foram apresentadas análises qualitativas ou quantitativas como, por exemplo, metas de comunicação, que permitam comprovar que as ações do plano anual de comunicação são de fato eficazes na divulgação da marca e dos produtos;

d. Avaliação do nível de conhecimento a respeito da marca e dos produtos - não há evidência de prática de gestão para a avaliação do nível de conhecimento dos clientes a respeito da marca e dos produtos dos Correios.

No Quadro 3 são evidenciados os indicadores usados pelos correios para mensurarem o capital intelectual. 


\begin{tabular}{|l|l|}
\hline CAPITAL INTELECTUAL & INDICADORES DOS CORREIOS \\
\hline \multirow{5}{*}{ CAPITAL HUMANO } & Funcionários por cargo \\
\hline Funcionários por nível de escolaridade \\
\hline Funcionários por faixa etária \\
\hline Absenteísmo por mês \\
\hline Educação e desenvolvimento por hora \\
\hline Despesa de pessoal pela despesa total \\
\hline Custo de Assistência médica por funcionário \\
\hline Rotatividade de funcionário \\
\hline Crescimento profissional \\
\hline Admissões e demissões por mês \\
\hline Satisfação dos funcionários \\
\hline CAPITAL ESTRUTURAL & Qualidade do ambiente de trabalho \\
\hline Condições de trabalho \\
\hline Atuação da chefia \\
\hline Qualidade na comunicação \\
\hline Favorabilidade da imagem institucional para o funcionário \\
\hline Integração do funcionário à empresa \\
\hline Favorabilidade do relacionamento entre as áreas \\
\hline Rotatividade de máquinas e equipamentos \\
\hline Investimento de recursos por funcionários \\
\hline Desempenho operacional \\
\hline Qualidade operacional \\
\hline Projetos desenvolvidos pelo Inova Correios \\
\hline Atendimento da CAC por mês \\
\hline Satisfação do cliente \\
\hline Satisfação de produtos e serviços \\
\hline Imagem dos Correios para os clientes \\
\hline Relacionamento do cliente com o concorrente \\
\hline Quantidade de cliente por segmentação do mercado \\
\hline Atendimento ao fornecedor por mês \\
\hline Demandas dos clientes pela internet - Fale com os Correios \\
\hline Captação de novos clientes por mês \\
\hline Agências de Correios por cliente \\
\hline
\end{tabular}

Figura 7 - Indicadores dos Correios relacionados à Capital Intelectual

Fonte: Dados da pesquisa

\subsection{Survey}

O survey foi aplicado por meio de um questionário encaminhado para 60 agências próprias dos Correios, sendo que na capital de São Paulo há cerca de 100 agências próprias. Contudo, foram escolhidas as que possuem maior fluxo de postagem e consequentemente maior número de clientes e maiores receitas de vendas. 
Do total de 60 questionários enviados para os gerentes das agências, 37 foram respondidos. Buscou-se com esta pesquisa compreender para cada indicador o grau de presença, a disponibilidade e a utilização.

Os quesitos "presença”, “importância" e "disponibilidade" foram avaliados numa escala de 1 à 5, sendo que quanto maior a nota melhor seria a classificação do indicador. Já o quesito "utilização" do indicador para a tomada de decisão foi avaliada através de 0 ou 1 , sendo 0 no caso de não usar o indicador e 1 para o caso de usar o indicador. Quando ocorreu empate nos resultados verificou-se as demais casas do valor total e persistindo o empate, considerou-se a ordem da presença do indicador.

O resultado consolidado da pesquisa é demonstrado na Tabela 1, onde os indicadores foram divididos nas três dimensões do capital intelectual. Os números apresentados referemse à média das respostas para cada indicador.

Tabela 1 - Resultado Consolidado do Estudo

\begin{tabular}{l|l|l|l|l}
\hline INDICADORES & QUESITOS & \multicolumn{2}{l}{} \\
\hline Capital Humano & Presença & Importância & Disponibilidade & Utilização \\
\hline Satisfação dos funcionários & 4,19 & 4,54 & 3,78 & 0,76 \\
\hline Desenvolvimento dos funcionários & 4,03 & 4,51 & 3,73 & 0,27 \\
\hline Valorização das habilidades & 3,57 & 4,54 & 3,54 & 0,54 \\
\hline Liderança e delegação & 3,54 & 4,24 & 3,35 & 0,51 \\
\hline Inovação & 3,24 & 4,16 & 3,19 & 0,38 \\
\hline Desligamento de funcionários & 2,49 & 3,35 & 2,89 & 0,14 \\
\hline Capital Estrutural & Presença & Importância & Disponibilidade & Utilização \\
\hline Eficiência nos processos & 4,35 & 4,59 & 4,19 & 0,89 \\
\hline Qualidade nos processos & 4,30 & 4,49 & 4,05 & 0,92 \\
\hline Gestão dos procedimentos & 4,08 & 4,46 & 3,86 & 0,70 \\
\hline Capacidade de atendimento & 3,89 & 4,27 & 3,70 & 0,54 \\
\hline Meio ambiente & 3,76 & 4,27 & 3,38 & 0,59 \\
\hline Sociedade em geral & 3,54 & 4,05 & 3,46 & 0,35 \\
\hline Aspectos econômicos e financeiros & 3,49 & 4,16 & 3,27 & 0,41 \\
\hline Estrutura organizacional & 3,46 & 3,86 & 3,16 & 0,38 \\
\hline
\end{tabular}




\begin{tabular}{l|l|l|l|l}
\hline Capital Relacional & Presença & Importância & Disponibilidade & Utilização \\
\hline Clientes dos Correios & 4,54 & 4,57 & 3,97 & 0,84 \\
\hline Gestão da carteira & 4,49 & 4,62 & 4,11 & 0,70 \\
\hline Canais de distribuição & 4,00 & 4,24 & 3,70 & 0,54 \\
\hline Quantidade de agências & 3,92 & 4,22 & 3,84 & 0,46 \\
\hline Qualidade do relacionamento & 3,76 & 4,22 & 3,54 & 0,49 \\
\hline Análise da concorrência & 3,65 & 4,43 & 3,16 & 0,32 \\
\hline Participação de mercado & 3,51 & 4,24 & 3,38 & 0,32 \\
\hline Despesas de atendimento & 3,27 & 4,11 & 3,11 & 0,27 \\
\hline Fon & & & & \\
\hline
\end{tabular}

Fonte: Dados da pesquisa

Tendo por início a análise a partir do Capital Humano, observa-se que os indicadores que tratam da satisfação do funcionário são os que possuem maior presença $(4,19)$, apresentam os maiores níveis de informações disponíveis $(3,78)$ e são os mais utilizados $(0,76)$. Estes indicadores também foram considerados pelos respondentes, juntamente com aqueles relacionados à valorização das habilidades, como os mais importantes $(4,54)$. Ainda sobre esta dimensão do capital intelectual, os indicadores que medem o desligamento de funcionários são os menos presentes $(2,49)$. Estes indicadores também são, na visão dos gerentes, os menos importantes $(3,35)$, os que possuem menor disponibilidade de informação $(2,89)$ e pouco utilizados na tomada de decisão $(0,14)$.

Com relação ao bloco de indicadores do Capital Estrutural, observa-se que aqueles que tratam da eficiência nos processos foram os mais presentes nas respostas analisadas $(4,35)$. Também foram considerados os mais importantes neste bloco $(4,59)$ e se apresentaram como os indicadores com maior nível de informações disponíveis $(4,19)$. Todavia, no que se refere à utilização, os indicadores relacionados à qualidade nos processos apareceram em primeira posição $(0,92)$. Continuando a análise sobre o Capital Estrutural, os indicadores que medem a estrutura organizacional foram os menos presentes $(3,46)$, considerados os de menor importância $(3,86)$ e com menos disponibilidade de informação $(3,16)$. Contudo, os indicadores menos utilizados neste bloco são aqueles relacionados à sociedade em geral $(0,35)$.

Por fim, no que se refere ao Capital Relacional, observa-se que os indicadores que tratam dos clientes dos Correios são os que possuem maior presença $(4,54)$ e são os indicadores mais utilizados $(0,84)$ pelos respondentes da pesquisa. Já os indicadores mais importantes neste bloco são os relacionados à gestão de carteira de clientes $(4,62)$., os quais também apresentaram maior nível de informações disponível $(4,11)$. Por outro lado, os 
indicadores que medem as despesas de atendimento são os menos presentes $(3,27)$, considerados de menor importância $(4,11)$, com menos disponibilidade de informação $(3,11)$ e menos utilizados na tomada de decisão $(0,27)$.

\subsection{Discussão dos Resultados}

No que se refere ao Capital Humano, 'desenvolvimento de funcionários' apresentou grande presença e importância nos questionários respondidos, corroborando os resultados de Andreeva e Garanina (2016), as quais identificaram alta relação deste item com a performance de empresas russas, e os achados de Ramírez, et al. (2015) que identificaram a relevância deste quesito no contexto de universidades públicas espanholas.

Quanto ao Capital Estrutural, 'qualidade nos processos' foi considerado importante para os gestores e bastante presente nos questionários, similar aos resultados de Ramírez, et al. (2015) que identificaram a gestão da qualidade como algo fundamental no setor de ensino na Espanha. Ainda com relação a esta dimensão, 'eficiência nos processos', o quesito mais presente e mais importante neste estudo teve grande influência no crescimento das vendas e do valor de mercado de empresas indianas, conforme Smriti e Das (2018).

Por fim, no Capital Relacional, o item 'cliente dos Correios' foi o mais presente na survey realizada e considerado um dos mais importantes pelos respondentes. O estudo de Ramírez, et al. (2015), ambientado em universidades públicas espanholas, apresentou resultados semelhantes, na medida em que o estudante é considerado um dos itens mais relevantes do Capital Relacional.

\section{CONCLUSÕES}

Um dos grandes desafios da empresa é a gestão eficiente do seu capital intelectual. Assim, este estudo teve como problema de pesquisa: como os Correios realizam a gestão do capital intelectual? Dessa forma, a pesquisa teve como objetivo evidenciar como os gerentes dos Correios gerem seu capital intelectual.

Para tal, a pesquisa contou com duas etapas: análise documental e pesquisa survey. Verificando a análise documental foi possível constatar alguns fatores que são fundamentais para uma empresa gerir o seu capital intelectual; dentre eles, destacam-se alguns pontos fortes observados na empresa analisada: plano de cargos e salários ajustado com a política de 
desenvolvimento profissional, criação do programa Inova Correios, e identificação das necessidades e expectativas dos clientes. Nesta etapa do estudo também foram constatadas algumas oportunidades de melhoria: não é possível avaliar a efetividade no processo de seleção e contratação de pessoas, a prática de benchmarking de processos não está sistematizada, e não há evidência para mensuração do nível de conhecimento dos clientes sobre a marca e produtos dos Correios.

Já na etapa da pesquisa survey, constatou-se que alguns indicadores são fundamentais na rotina da empresa, tanto pela presença quanto pela importância; são eles: satisfação do funcionário, eficiência nos processos, qualidade nos processos, clientes dos Correios e gestão da carteira de clientes. Por outro lado, alguns indicadores obtiveram médias baixas e podem indicar necessidade de atenção maior por parte dos gestores da empresa: desligamento de funcionários, estrutura organizacional, sociedade em geral e despesas de atendimento.

Dessa forma, após a análise documental e a survey conclui-se que os gestores dos Correios utilizam indicadores para mensurar o capital intelectual da empresa em suas três dimensões. Além disso, pode-se afirmar que a empresa vem aprimorando a forma como mede o seu ativo intangível, dado que em 2010 a companhia implantou o modelo de gestão do PNQ para aperfeiçoar a mensuração do capital intelectual.

Vale destacar que tais resultados não podem ser generalizados para outras agências dos Correios as quais não fizeram parte da pesquisa. Sugere-se, então, estender o estudo não somente para outras agências do Estado de São Paulo, mas também para agências de outros estados do Brasil, com o intuito de identificar diferentes tendências de gestão do capital intelectual em distintas regiões do país. O estudo também pode ser replicado em outras empresas do segmento para verificar a similaridade ou não das conclusões aqui encontradas.

\section{REFERÊNCIAS}

Andrade, M.M. (2010). Introdução à Metodologia do Trabalho Científico. São Paulo. Atlas.

Andreeva, T., \& Garanina, T. (2016). Do all elements of intellectual capital matter for organizational performance? Evidence from Russian context. Journal of Intellectual Capital, 17(2), 397-412.

Antunes, M. T. P. \& Martins, E. (2002). Capital intelectual: verdades e mitos. Revista Contabilidade \& Finanças, 13(29), 41-54.

Arnosti, J. C. M, Neumann, R. A., Oliveira, E.C.M. \& Yomura, N. (2009). Capital Intelectual: Reconhecimento e Mensuração. São Paulo: Juruá. 
Bervian, P.A; Cervo, A.L; Da Silva, R. (2007). Metodologia Científica. São Paulo: Pearson.

Bollen, L., Vergauwen, P., \& Schnieders, S. (2005). Linking intellectual capital and intellectual property to company performance. Management Decision, 43(9), 1161-1185.

Bontis, N. (1998). Intellectual capital: an exploratory study that develops measures and models. Management decision, 36(2), 63-76.

Carson, E., Ranzijn, R., Winefield, A., \& Marsden, H. (2004). Intellectual capital: Mapping employee and work group attributes. Journal of intellectual capital, 5(3), 443-463.

Contador, J. C., Contador, C. A., de Carvalho, M. F. H., \& Contador, J. L. (2006). Metodologia para análise e ampliação da competitividade empresarial. Ciencias da Administraçao, 8(15), 5 .

Correios. (2013). Relatórios da Administração dos Correios: Exercício 2012. Recuperado em 15 de ago de 2013, de http://www.correios.com.br/sobreCorreios/empresa/publicacoes/arquivos_pdf/RelatorioAdm inistracao_2012.pdf.

Demo, G., Fogaça, N., Nunes, I., Edrei, L., \& Francischeto, L. (2011). Políticas de gestão de pessoas no novo milênio: cenário dos estudos publicados nos periódicos da área de administração entre 2000 e 2010. RAM. Revista de Administração Mackenzie, 12(5), 15-42.

Dias, C. M. Jr, Lima, M. V. A., \& Dalmau, M. B. L. (2011). Uma contribuição ao desenvolvimento de indicadores de desempenho para ativos intangíveis organizacionais. Revista de Ciências da Administração, 13(31), 11-29.

Dumay, J., Rooney, J., \& Marini, L. (2013). An intellectual capital-based differentiation theory of innovation practice. Journal of Intellectual Capital, 14(4), 608-633.

Edvinsson, L. \& Malone, M.S. (1998). Capital intelectual: Descobrindo o valor real de sua empresa pela identificação de seus valores internos. São Paulo: Makron Books.

Estrada, R. J. S., \& de Almeida, M. I. R. (2007). A eficiência e a eficácia da gestão estratégica: do planejamento estratégico à mudança organizacional. Revista de ciências da administração, 9(19), 147-178.

Fontenelle, I. A. (2012). Para uma crítica ao discurso da inovação: saber e controle no capitalismo do conhecimento. RAE-Revista de Administração de Empresas, 52(1), 100-108.

Fundação Nacional Da Qualidade (FNQ). (2007). Capital intelectual e inovação: a gestão dos intangíveis. São Paulo, 2007.

Galbreath, J. (2002). Twenty-first century management rules: the management of relationships as intangible assets. Management Decision, 40(2), 116-126.

Gião, P. R., \& Oliveira, M. D. M. Jr. (2009). Offshoring de serviços de call center: um estudo 
comparativo entre Brasil, Índia e África do Sul. REGE Revista de Gestão, 16(2), 17-32.

Gibbert, M., Leibold, M., \& Voelpel, S. (2001). Rejuvenating corporate intellectual capital by co-opting customer competence. Journal of Intellectual Capital, 2(2), 109-126.

Gracioli, C., Godoy, L. P., Lorenzett, D. B., \& Godoy, T. P. (2012). Capital intelectual: uma ferramenta inovadora na busca por vantagens competitivas. RAI Revista de Administração e Inovação, 9(4), 96-120.

Iudícibus, S. (1997). Teoria da Contabilidade. São Paulo, Atlas.

Kaplan, R. S.; Norton, D. P. (1992). The balanced scorecard - measures that drive performance. Harvard Business Review, 71-79.

Khalique, M., Bontis, N., Shaari, J. A. N., \& Isa, A. H. Md. (2015). Intellectual capital in small and medium enterprises in Pakistan. Journal of Intellectual Capital, 16(1), 224-238.

Khan, S. (2011). The interactive effects of intellectual capital components on the relevance of the balance sheet as an indicator of corporate value. Journal of American Academy of Business, 130-136.

Kich, J. I. F., Pereira, M. F., Emmendoerfer, M. L., \& dos Santos, A. M. (2008). A influência do líder na condução do processo de implementação do planejamento estratégico. Ciencias da Administraçao, 10(21), 122-146.

Krücken-Pereira, L., de Abreu, A. F., \& Bolzan, A. (2002). A necessidade de inovar: um estudo na indústria de alimentos. Revista de Ciências da Administração, 4(6), 19-27.

Lev, B. 2001. Measuring the value of Intellectual Capital. Ivey Business Journal, 65(4):1620.

Lima, A. D. A., \& Baptista, P. D. P. (2013). Impacto da congruência entre autoconceito e personalidade de marca na intensidade da qualidade de relacionamento e lealdade do consumidor. Revista Brasileira de Marketing, 12(1), 73-96.

Matos, B., Reina, D., Ensslin, S.R. \& Reina, D.R.M; (2011). Evidenciação voluntária do capital intelectual de natureza social e ambiental da empresa Natura Cosméticos S.A. no período entre 2003 e 2008. Revista Contemporânea de Economia e Gestão, 9, (1),67-77.

Oliveira, A.B.S. (1999). Contribuição à formulação de um modelo decisório para intangíveis por atividade: uma abordagem de gestão econômica. São Paulo. 1999. Tese Doutorado. Faculdade de Economia, Administração e Contabilidade da Universidade de São Paulo.

Olve, N., Roy, J. \& Wetter, M. (1999). Performance drivers: a practical guide to using the Balanced Scorecard. London, John Wiley \& Sons.

Piva, L. C., Fumagalli, L. A. W., de Paula Baptista, P., \& da Silva, W. V. (2007). Relação entre satisfação, retenção e rentabilidade de clientes no setor de planos de saúde. Revista de Ciências da Administração, 9(19), 54-80. 
Ramírez, Y., Manzaneque, M., \& Priego, A. M. (2015). Formulating and elaborating a model for the measurement of intellectual capital in Spanish public universities. International Review of Administrative Sciences, 83(1), 149-176.

Reis, A., \& Giacomini, G. F (2008). Indicadores de Responsabilidade Social: estudo comparativo entre empresas públicas e privadas, baseado no Balanço Social IBASE. Revista de Ciências da Administração, 10(22), 171-185.

Rezende, J. F. C., Avila, M., \& Maia, R. S. (2012). Geração e gestão do valor por meio de métricas baseadas nas perspectivas do capital intelectual. Revista de Administração, 47(1), 51-67.

Rozzett, K., \& Demo, G. (2010). Desenvolvimento e validação fatorial da escala de relacionamento com clientes (ERC). RAE-Revista de Administração de Empresas, 50(4), 383395.

Scherer, F. L., \& Gomes, C. M. (2010). Internacionalização De Empresas: Perspectivas Estratégicas No Setor Coureiro-Calçadista. REGE Revista de Gestão, 17(1), 27-44.

Schneider, C., \& Moretto Neto, L. (2006). Avaliação do desempenho organizacional da empresa familiar Indústrias Alfa SA* com base no modelo de gestão estratégica balanced scorecard. REGE Revista de Gestão, 13(1), 89-104.

Secundo, G., Dumay, J., Schiuma, G., \& Passiante, G. (2016). Managing intellectual capital through a collective intelligence approach: an integrated framework for universities. Journal of Intellectual Capital, 17(2), 298-319.

Silva, G. A. V., Basques, P. V., \& Facco, F. C. (2006). Conectando recursos humanos e estratégia: em busca de indicadores de resultados na gestão de pessoas. REGE Revista de Gestão, 13(3), 87-100.

Silva, J. F, F'., Holanda, L. M. C. \& Rocha, R.C.C. (2006). Capital intelectual: Um estudo de caso numa empresa de jornalismo. In: XIII SIMPEP - Bauru, SP, Brasil, 6 a 8 de Novembro de 2006.

Smriti, N., \& Das, N. (2018). The impact of intellectual capital on firm performance: a study of Indian firms listed in COSPI. Journal of Intellectual Capital, 19(5), 935-964.

Souza, A, A., Guerra, M., Lara, C. O., Gomide, P. L. R., Pereira, C. M., \& Freitas, D. A. (2009). Controle de gestão em organizações hospitalares. REGE Revista de Gestão, 16(3), 1529.

Stewart, T.A. (1998). Capital intelectual: a nova vantagem competitiva das empresas. Rio de Janeiro, Editora Campus.

Vasconcelos, S.P. (2010). Decisões estratégicas de localização de supermercados: um estudo de caso. Revista Brasileira de Economia de Empresas, 10(1), 51-62. 
Vieira, V. A., Appio, J., \& Slongo, L. A. (2007). Uma análise dos antecedentes da satisfação no trabalho em uma empresa madeireira. REGE Revista de Gestão, 14(3), 1-18.

Vital, J. T., Cavalcanti, M. M., Dalló, S., de Oliveira Moritz, G., \& Costa, A. M. (2009). A influência da participação no Índice de Sustentabilidade Empresarial (ISE) no Desempenho Financeiro das empresas. Revista de Ciências da Administração, 11(24), 11-40.

Wernke, R., Lembeck, M. \& Bornia, A. C. (2003). As considerações e comentários acerca do capital intelectual. Revista da FAE, 6(1). 